\title{
Cosmological constant and the fate of the DDM theory
}

\author{
M. M. Ćirković ${ }^{1}$ and S. Samurović ${ }^{2}$ \\ 1 Astronomical Observatory, Volgina 7, 11000 Belgrade, Yugoslavia \\ 2 Dipartimento di Astronomia, Università di Trieste, Via Tiepolo 11, 34131 Trieste, Italy \\ e-mail: srdjan@ts.astro.it
}

Received 3 April 2001 / Accepted 2 May 2001

\begin{abstract}
We investigate the impact of the non-zero cosmological constant on the classical decaying dark matter theory developed by the late Dennis Sciama. In particular, we concentrate on the change in relevant values of cosmological parameters in comparison to the high-precision estimates given by Sciama (1997). It is shown that the appropriate changes in resulting parameter values are such to make the DDM concept less plausible. This is in complete agreement with recently reported observational results detrimental to this theory.
\end{abstract}

Key words. elementary particles - cosmology: dark matter - cosmology: diffuse radiation - distance scale

\section{Introduction}

Decaying dark matter (DDM) theory is an attempt to simultaneously solve two important problems of contemporary astrophysics: the dark matter problem in spiral galaxies, like the Milky Way, and the problem of ionization of the interstellar and intergalactic medium (Sciama 1993). To achieve these goals, theory introduces massive decaying neutrino with the mass $m_{\nu} \sim 30 \mathrm{eV}$. This neutrino has a decay lifetime of $2 \pm 1 \times 10^{23} \mathrm{~s}$, that produces a decay photon of energy of $13.7 \pm 0.1 \mathrm{eV}$ (Sciama 1998). This theory is heavily constrained, i.e. its parameters are very well defined, with extremely small uncertainties. An experiment, EURD, has been proposed in order to test the theory (Sciama 1993). Results recently published suggest that this theory is no longer viable, because the emission predicted by the DDM theory was not registered (Bowyer et al. 1999). In this Paper we wish to investigate the values of the Hubble constant and predicted age of the universe in the DDM theory, in the light of two recent important empirical discoveries: first that neutrinos do have mass (Fukuda et al. 1998), and the second one according to which there exists a large positive cosmological constant (Perlmutter et al. 1998, 1999; Reiss et al. 1998).

In this respect, it seems that we are in the middle of a major change of cosmological paradigm (not unexpected, however, as even the cursory look at the relevant literature could show). Recent results of the surveys of the type I supernovae at cosmological distances indicate the possible presence of a large cosmological constant (Perlmutter

Send offprint requests to: M. M. Ćirković, e-mail: mcirkovic@aob.aob.bg.ac.yu et al. 1998, 1999; Reiss et al. 1998). If the total cosmological density parameter corresponds to the flat $(\Omega=1)$ universe, the contribution due to matter density is (total $1 \sigma$ statistical + systematic errors quoted)

$\Omega_{\mathrm{m}}=0.28_{-0.12}^{+0.14}$.

This result suggests not only that the universe will expand indefinitely, but that it will expand in an (asymptotically) exponential manner, similar to the early inflationary phase in its history. In addition to these observations, we use results from the primordial nucleosynthesis which are entering the high-precision phase (Schramm \& Turner 1998), and limit the combination of baryonic density fraction and the Hubble parameter. We shall use the following (conservative) limits:

$\Omega_{\mathrm{b}} h^{2}=0.025 \pm 0.005$.

These are larger values than those used by Sciama (1997), but this can be justified on several counts. First of all, later measurements of deuterium abundance at high redshift unambiguously indicate lower abundances than previous controversial values (Burles \& Tytler 1998). In addition, measurements of HeII Gunn-Peterson effect at high redshift (Jakobsen 1998) gave very high values for $\Omega_{\mathrm{b}} h^{2}$, even higher than those in Eq. (2). For the sake of completeness, we have used both this realistic, and the lower value of Sciama (1997) in further calculations.

One should add the following epistemological consideration. Being the property of the quantum vacuum itself, addition of the non-zero cosmological constant does not prima facie increase the conceptual complexity of the theory for dark matter. However, if we believe in classical prediction of the inflationary scenario $\Omega=1 \pm \epsilon$ with 
the precision $\epsilon \simeq 10^{-5}$, we have to take into account this additional constraint on the distribution of total energy density in the universe. We shall use this assumption in the further considerations.

We shall use the following notation: symbol $\Omega$ without any subscripts will be reserved for the total density parameter of the universe, which, according to our present understanding can be written as the sum of densities of matter $^{1}$ and vacuum density (which is manifested in the form of the cosmological constant $\Lambda$ ), i.e.

$\Omega \equiv \Omega_{\mathrm{m}}+\Omega_{\Lambda}$

The contribution of matter can be written as

$\Omega_{\mathrm{m}} \equiv \frac{\rho_{\mathrm{m}}}{\rho_{\text {crit }}}=\frac{8 \pi G \rho_{\mathrm{m}}}{3 H_{0}^{2}}$

and the one of the cosmological constant as

$\Omega_{\Lambda}=\frac{c^{2} \Lambda}{3 H_{0}^{2}}=2.8513 \times 10^{55} h^{-2} \Lambda$,

$\Lambda$ being in units of $\mathrm{cm}^{-2}$. The present day Hubble constant is parametrized in a standard way as $H_{0} \equiv$ $100 \mathrm{hm} \mathrm{s}^{-1} \mathrm{Mpc}^{-1} . \Lambda$ enters the Einstein field equations as

$R_{\mu \nu}-\frac{1}{2} g_{\mu \nu} R-\Lambda g_{\mu \nu}=-\frac{8 \pi G}{c^{4}} T_{\mu \nu}$,

and $\Lambda$-universes are the homogeneous and isotropic solutions of these tensor equations (for other notation see any of the standard General Relativity textbooks, e.g. Weinberg 1972; for history and phenomenology of the cosmological constant, see the detailed review of Carroll et al. 1992, and references therein). We now wish to investigate whether an inflationary DDM universe can be reconciled with non-zero $\Lambda$ and still perform its explanatory tasks.

\section{Simple parameter estimates}

It is not possible to proceed in as simple way as in Sciama (1997), since the combination $\Omega_{\Lambda} h^{2}$ does not have an obvious physical meaning. However, we shall use this circumstance in order to establish plausible values for $h$ first. For establishing connection between ionizing flux $F$ and $m_{\nu}$ we follow the same simple procedure outlined by Sciama (1997), except that it is not possible any more to simply plug in the "final" value for $h$ as it has been done in that study. This situation gives rise to the term linear in $h$, which is the main source of difficulties here. Therefore, we obtain for the decaying neutrino mass

$m_{\nu}=(27.2+0.39 h) \pm 0.39 h \mathrm{eV}$.

Hence, the contribution to the cosmological density fraction is

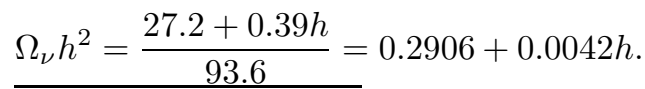

\footnotetext{
1 Including presently negligible contribution of radiation $\Omega_{\mathrm{rad}}=4.31 \times 10^{-5} h^{-2}$.
}

Assuming in the spirit of DDM theory that $\Omega_{\mathrm{m}}=\Omega_{\nu}+\Omega_{\mathrm{b}}$ we can write

$\Omega h^{2}=\Omega_{\Lambda} h^{2}+\Omega_{\nu} h^{2}+\Omega_{\mathrm{b}} h^{2}$

or, equivalently, taking into account Eqs. (2) and (8), we have

$$
\begin{aligned}
\left(\Omega-\Omega_{\Lambda}\right) h^{2} & =0.025+0.2906+0.0042 h \\
& =0.3156+0.0042 h .
\end{aligned}
$$

Now we may use the theoretical prejudice for $\Omega=1$ that is in agreement with the recent Boomerang result (de Bernardis et al. 2000), and therefore $\Omega_{\Lambda}$ is determined by Eq. (1). Later we shall discuss the consequences of variations in $\Omega$ in the observationally allowed range (approximately $0.3-1.1$ ). The physically acceptable solution of this quadratic equation in $h$ is $h=1.07$.

The physical picture here is highly intuitive: for the fixed total density parameter, introduction of a term with negative effective pressure in the Friedmann equation results in a faster expansion rate. However, all recent observational measurements have suggested lower values for the Hubble parameter, in the $0.5-0.8$ range, even tending toward the lower limit (e.g. Paturel et al. 1998; Schaefer 1998). We notice that we recover the particular value $h=0.55$ for $\Lambda=0$ obtained by Sciama (1997), as expected.

\section{Discussion}

The age of the universe predicted in such any theory with non-zero $\Lambda$ is given as (e.g. Weinberg 1972; Carrol et al. 1992)

$t_{0}=\frac{1}{H_{0}} \int_{0}^{1} \mathrm{~d} x\left[\left(1-\Omega_{\mathrm{m}}-\Omega_{\Lambda}\right)+\Omega_{\mathrm{m}} x^{2-3(1+\alpha)}+\Omega_{\Lambda} x^{2}\right]^{-\frac{1}{2}}$

Here $\alpha$ defines the equation of state of the present matter, being effectively the ratio of pressure to energy density. For $\Omega=1$ case, this reduces to the well-known relation (e.g. Singh 1995)

$t_{0}=\frac{2}{3 H_{0}} \frac{1}{\sqrt{\Omega_{\Lambda}}} \ln \left[\frac{1+\sqrt{\Omega_{\Lambda}}}{\sqrt{1-\Omega_{\Lambda}}}\right]$.

In Fig. 2 we present the age estimates for the same two cases as in Fig. 1 in the $\Lambda+$ DDM theory. This is to be compared with the best estimate of the current age of the universe for $\Omega_{\Lambda}$ given by Eq. (1) is (Perlmutter et al. 1999)

$t_{0}=14.9_{-1.1}^{+1.4} \times \frac{0.63}{h} \mathrm{Gyr}$.

In addition, it should be compared to the age of globular clusters recently carefully measured with accounting for the revised Hipparcos distance scale (Chaboyer et al. 1996, 1998). We perceive that the estimates presented in Fig. 2 are rather significantly smaller from the result in Eq. (13), although they have a correct correspondence limit $\Lambda=$ 0 of $t_{0} \approx 12 \mathrm{Gyr}$ as in Sciama (1997). So low ages are inconvenient from the point of view of globular cluster 


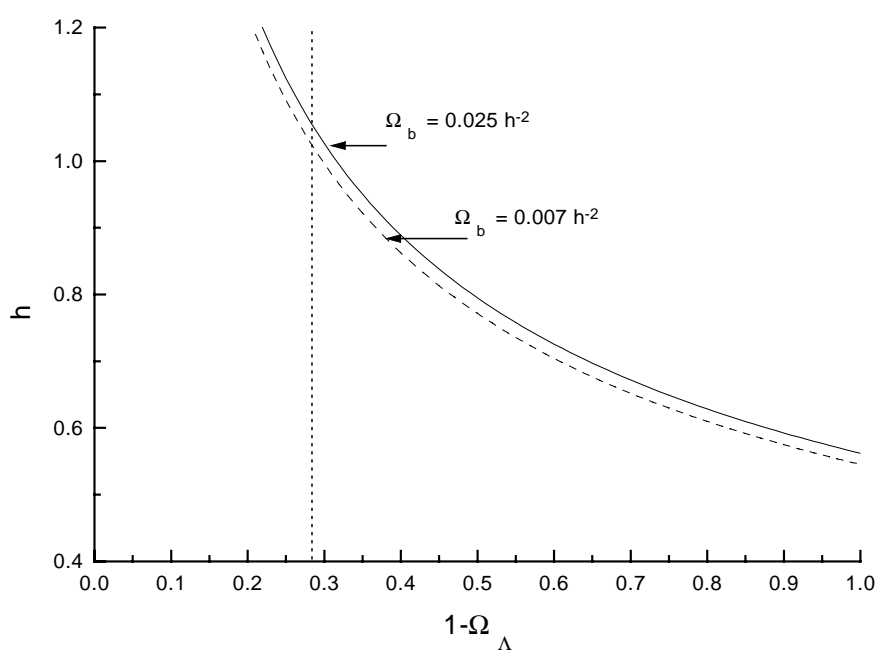

Fig. 1. The value of the Hubble parameter, $h$, as a function of the cosmological constant $\Lambda$, i.e. its contribution to the total cosmological density (assumed to be unity). For the sake of clarity, we plotted $1-\Omega_{\Lambda}$ which represents contribution of the neutrinos and baryonic matter to the total cosmological density. Vertical dotted line, plotted at $1-\Omega_{\Lambda} \approx 0.28$ corresponds to the realistic contribution of matter in the universe. It is obvious that in this case $h$ tends to the unrealistically high value of $h \sim 1$. The different possible contributions of the baryonic matter to the total cosmological density are represented by two curves: solid (realistic higher $\Omega_{\mathrm{b}}$, see text) and dashed (used by Sciama 1997).

ages, as well as our understanding of the most distant galaxies observed.

Considering the current trend in observational estimates of cosmological parameters, the impact of cosmological constant on parameter values in DDM theory is largely negative. Resulting values of corrected parameters for $\Lambda \neq 0$ version of Sciama's theory make the entire scheme less plausible. In that respect, recent results of the EURD mission are highly indicative of the observational verdict. This mission failed to observe the emission of the dark sky at wavelengths slightly lower than $912 \AA$, with the limit ( $95 \%$ confidence) of only a third of the predicted intensity.

The negative EURD result is not the only indication of problems of the DDM theory. Recently, Maloney \& BlandHawthorn (1999) calculated the flux from a full-neutrino halo and obtained: $\phi \approx 2-3 \times 10^{5}$ photons $\mathrm{cm}^{-2} \mathrm{~s}^{-1}$. They find that the observed emission is much fainter: $\phi \sim 10^{4}$ photons $\mathrm{cm}^{-2} \mathrm{~s}^{-1}$. The detrimental consequences of this result for DDM cannot be remedied by introduction of $\Lambda$, since the latter does not impact galactic dynamics, and therefore the estimates of necessary amount of dark matter in galactic haloes. Of course, one could always assert that DDM is only a small $(\sim 10 \%)$ part of the dark halo, most of it being in the form of baryons (i.e. MACHOs and/or molecular clouds; see Fields et al. 1998; Samurović et al. 1999, also Sciama 2000). In that version, DDM is dominant only on larger than galactic scales. However, the entire rationale of the theory is undermined in this

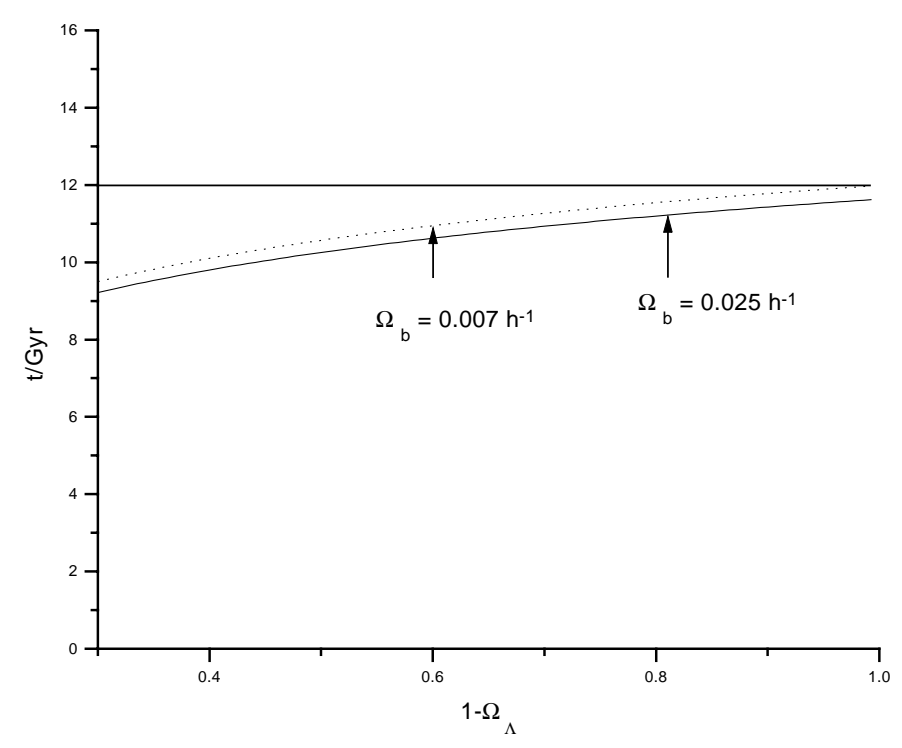

Fig. 2. The ages predicted by $\Lambda+$ DDM flat cosmological model. The same notation as in Fig. 1 is used.

way, since there is no more any direct connection between cosmology and the ISM physics, and the properties of the decaying neutrino can not be constrained with remarkable precision any more. The same criticism applies to the open DDM models (i.e. $\Omega_{\nu} \approx \Omega<1$ ) which requires even more fine-tuning, in particular in view of the consequent properties of matter in galaxy clusters.

However, even the fine-tuned version of the theory fails if confronted with negative results in particle experiments on neutrino masses. Although recent results on the oscillations of atmospheric neutrinos (Fukuda et al. 1998) are sensitive only to the mixing angles and mass difference $\Delta m^{2}$ between the two neutrino flavors, the results are somewhat indicative in suggesting rather low, probably sub-eV neutrino masses. Although the DDM theory was correct in assuming neutrino masses - the first empirical result in particle physics outside of the Standard Model - only experiments currently in progress will show whether the required neutrino masses are compatible with empirical limits.

Obviously, the simplicity and elegance of the original DDM theory is lost with any complication such as discussed in the present paper. Any attempt of bringing it in accordance with the observational data must result, it seems, in more and more contrived versions of the original beautiful idea. In this sense, we may compare it with the classical steady state cosmological model of Bondi \& Gold (1948), as well as Hoyle (1948), which has been discredited in the course of progress of observations, but which has had an epochal impact on the very formation of modern cosmology (Kragh 1996). In the same manner, Sciama's DDM theory, although it may be regarded as disproved by now, has inspired and provoked an immense theoretical and observational activity in astrophysics and cosmology. The results of these efforts will certainly present its lasting legacy. 
Acknowledgements. The authors wholeheartedly thank Vesna Milošević-Zdjelar for help in finding several important references. S.S. acknowledges the financial support of the Abdus Salam International Centre for Theoretical Physics, Trieste. This research has made use of NASA's Astrophysics Data System Abstract Service.

\section{References}

Bondi, H., \& Gold, T. 1948, MNRAS, 108, 252

Bowyer, S., Corpela, E. J., Edelstein, J., et al. 1999, ApJ, 526, 10

Burles, S., \& Tytler, D. 1998, ApJ, 499, 699

Carroll, S. M., Press, W. H., \& Turner, E. L. 1992, ARA\&A, 30, 499

Chaboyer, B., Demarque, P., Kernan, P. J., Krauss, L. M., \& Sarajedini, A. 1996, MNRAS, 283, 683

Chaboyer, B., Demarque, P., Kernan, Peter, J., \& Krauss, L. M. 1998, ApJ, 494, 96

de Bernardis, et al. 2000, Nature, 404, 955

Fields, B. D., Freese, K., \& Graff, D. S. 1998, New Ast., 3, 347

Fukuda, Y., et al. 1998, Phys. Rev. Lett., 81, 1562

Hoyle, F. 1948, MNRAS, 108, 372

Jakobsen, P. 1998, A\&A, 331, 61
Kragh, H. 1996, Cosmology and Controversy (Princeton University Press, Princeton)

Maloney, P. R., \& Bland-Hawthorn, J. 1999, in Stromlo Workshop on High-Velocity Clouds, ed. B. K. Gibson, \& M. E. Putman, ASP Conf. Ser., 166, 199

Paturel, G., Lanoix, P., Teerikorpi, P., et al. 1998, A\&A, 339, 671

Perlmutter, S., Aldering, G., Della Valle, M., et al. 1998, Nature, 391, 51

Perlmutter, S., et al. 1999, ApJ, 517, 565

Reiss, A. G., Filippenko, A. V., Challis, P., et al. 1998, AJ, 116,1009

Samurović, S., Ćirković, M. M., \& Milošević-Zdjelar, V. 1999, MNRAS, 309, 63

Schaefer, B. E. 1998, ApJ, 509, 80

Schramm, D. N., \& Turner, M. S. 1998, Rev. Mod. Phys., 70, 303

Sciama, D. W. 1993, Modern Cosmology and the Dark Matter Problem (Cambridge University Press, Cambridge)

Sciama, D. W. 1997, MNRAS, 289, 945

Sciama, D. W. 1998, A\&A, 335, 12

Sciama, D. W. 2000, MNRAS, 312, 33

Singh, A. 1995, Phys. Rev. D, 52, 6700

Weinberg, S. 1972, Gravitation and Cosmology (Wiley, New York) 